Костановський В. В., Мачалін І.О.

\title{
РАЗРАБОТКА И ИССЛЕДОВАНИЕ УНИВЕРСАЛЬНЫХ МОДЕЛЕЙ НАДЕЖНОСТИ АКТИВНОЙ ФАЗИРОВАННОЙ АНТЕННОЙ РЕШЕТКИ МНОГОФУНКЦИОНАЛЬНОЙ РАДИОЛОКАЦИОННОЙ СТАНЦИИ
}

В роботі проведено короткий аналіз моделей надійності АФАР, запропонованими вченими i інженерами за останні 20 років. Розглянуто модель надійності приймальні АФАР за критерієм допустимого підвищення мінімального рівня бічних пелюсток діаграми спрямованості амплітудно-фазового розподілу антеною решітки. Проаналізовано модель надійності передавальної АФАР за критерієм допустимого зниження максимальної дальності РЛС. Показано, щзо розглянуті моделі надійності $\epsilon$ наближеними (використовують експонентний закон розподілу відмов АФАР) $i$ тому можуть використовуватися тільки для наближеного визначення середнього напрацювання до відмови антеною решітки. Для визначення імовірнісних показників надійності АФАР: ймовірності безвідмовної роботи, інтенсивності відмов, щільності розподілу відмов, гаммавідсоткових ресурсу $і$ ін. Необхідні більш універсальні моделі надійності, щэо враховують структуру $i$ складність побудови антеною решітки як системи, щзо складається 3 величезної кількості основних $і$ резервних приймально передавальних каналів $i$ модулів. $У$ роботі розроблені і досліджені універсальні моделі надійності АФАР багатофункиіональної РЛС з дворівневої структурною схемою надійності (дворівнева АФАР). Універсальні моделі надійності враховує два основних фізичних критерію відмови АФАР: допустиме зниження максимального рівня дальності РЛС і припустиме збільшення мінімального рівня бічних пелюсток діаграми спрямованості антеною решітки. Як законів розподілу відмов випромінюючих (передавальних) $i$ прийомних каналів $i$ модулів антеною решітки застосовується експоненціальне розподіл (характеризує раптові відмови), немонотонний диффузионний розподіл (характеризує поступові відмови) $і$ композичія експоненціального $i$ дифузійного немонотонного закону розподілів (характеризує спільне прояв раптових $i$ поступових відмов) . У статті представлені рівняння і формули для визначення показників надійності передавальної, приймальні та приймально-передавальної АФАР: середнього напрацювання до відмови, імовірності безвідмовної роботи та ін.

В роботі розглянуто три ілюстративних прикладу розрахунку показників надійності передавальної, приймальні та приймально-передавальної АФАР при експоненційному розподілі відмов каналів $i$ модулів антеною решітки. Проведено аналіз впливу інтенсивностей відмов випромінюючих каналів і модулів джерел вторинного живлення на середній наробіток до відмови АФАР. Представлені в статті універсальні моделі надійності АФАР рекомендується використовувати при розробці нових багатофункиіональних РЛС з АФАР, а також можуть бути корисні студентам $i$ аспірантам університетів і вищих навчальних закладів в навчальному процесі.

Ключові слова: показники надійності, середнє напраџювання до відмови, активна фазована антенна решітка, передаючі і прийомні канали антеною решітки

\section{1. Введение}

Морские многофункциональные радиолокационные станции (РЛС) являются технически сложными, дорогостоящими радиоэлектронными системами. Они совмещают функции обнаружения и точного сопровождения целей с возможностью наведения большого количества зенитных управляемых ракет по выбранным целям. Особую роль в современной 
радиоэлектронике играют фазированные антенные решетки (ФАР). Технологии создания ФАР за последние 20-25 лет развиваются наиболее интенсивно. Их применение позволило увеличить скорость обзора пространства, улучшить характеристики антенных систем, обеспечить возможность многофункциональной работы РЛС различного назначения. Создание надежных радиолокационных средств с ФАР является долгосрочной целью и приоритетной задачей военно-технической политики. Ввиду расширения объема решаемых задач создаются качественно новые системы следующего поколения, развиваются технологии РЛС с активными ФАР (АФАР). В мире большое внимание уделяется разработке новых и модернизации состоящих на вооружении многофункциональных РЛС боевых кораблей. Более 30 лет на вооружении ВМС США находится корабельная многофункциональная радиолокационная станция AN/SPY-1. За это время разработано пять основных модификаций этой РЛС [1]. Широкое применение АФАР нашли в многофункциональных РЛС наземных зенитных ракетных комплексов США: Patriot (PAC-3), MEADS, THAAD. При этом АФАР многофункциональных РЛС могут включать в свой состав до несколько десятков тысяч активных сверхвысокочастотных (СВЧ) модулей. Учитывая, что АФАР многофункциональных РЛС являются дорогостоящими радиоэлектронными системами ответственного назначения с большим ресурсом и длительным сроком службы, то актуальной является проблема разработки методов расчета надежности.

\section{2. Краткий обзор}

Разработке моделей надежности АФАР посвящен ряд работ американских и отечественных исследователей [2-10]. Американскими учеными А. К. Агравалом и Э. Л. Хольцманом [2-4] рассматривалась модель надежности АФАР РЛС по критерию допустимого повышения минимального уровня боковых лепестков.

В работе [2] исследуется модель надежности приемной АФАР, представленной матрицей из 8000 элементов, со значением амплитуды главного бокового лепестка распределения Тейлора, равного минус 40 дБ, и гексагональной структурой размещения излучателей, расстояние между элементами которой составляет половину длины волны. Критерием отказа АФАР при этом считается повышение (выше допустимого уровня) минимального уровня ближних боковых лепестков диаграммы направленности амплитудно-фазового распределения антенной решетки. Отказ АФАР фиксируется при превышении допустимого количества отказов любого из трех видов компонентов антенной решетки: приемопередающих модулей, модулей управления и модулей источников вторичного электропитания. В работе [2] представлены значения предельно допустимых количеств отказов компонентов АФАР при повышении уровня боковых лепестков на $3 \mathrm{~dB}$ : $F_{T / R}=256, F_{C M}=5, F_{P S}=5$

и при п повышении уровня боковых лепестков на $6 \mathrm{~dB}: F_{T / R}=512, F_{C M}=10, F_{P S}=10$.

Предельные количества отказов компонентов АФАР:

приемо-передающих модулей $-F_{T / R}$, модулей управления - $F_{C M}$ и модулей источников питания - $F_{P S}$ определялись с использованием метода статистического моделирования уровня боковых лепестков диаграммы направленности амплитудно-фазового распределения антенной решетки.

В работе [2] представлена формула для определения средней наработки на отказ АФАР $\left(M T B F_{A N T}\right)$ :

$$
\operatorname{MTBF}_{A N T}=\frac{1}{\frac{N_{T / R}}{F_{T / R} M T B F_{T / R}}+\frac{N_{C M}}{F_{C M} M T B F_{C M}}+\frac{N_{P S}}{F_{P S} M T B F_{P S}}}
$$


Из формулы (1), можно вывести формулу для расчета интенсивности отказов АФАР:

$$
\lambda_{A N T}=\lambda_{T / R}+\lambda_{C M}+\lambda_{P S}
$$

где

$$
\lambda_{T / R}=\frac{N_{T / R}}{F_{T / R} M T B F_{T / R}}, \lambda_{C M}=\frac{N_{C M}}{F_{C M} M T B F_{C M}}, \lambda_{P S}=\frac{N_{P S}}{F_{P S} M T B F_{P S}} .
$$

Характер формулы (2) показывает, что для модели надежности АФАР РЛС в работе [ 2 ] используется экспоненциальное распределение отказов.

В работах $[5,6,7]$ рассматриваются модели надежности невосстанавливаемой ФАР с одноуровневой структурной схемой надежности (далее одноуровневая ФАР). В работе [5] рассматривается модель надежности невосстанавливаемой ФАР, структура которой включает: $N(t=0)=n+m$ СВЧ элементов, находящихся в начальный момент времени $(t=0)$ в работоспособном состоянии, в числе которых $n$ рабочих и $m$ - резервных элементов.

Критерием отказа невосстанавливаемой ФАР, является отказ $(m+1)$ - го СВЧ элемента в момент времени $t=T_{0_{-} A P A R}$, то есть выполнение условия 1 : $N\left(t=T_{0_{-} \text {АРАR }}\right)=N(t=0)-m-1$ или условия $2: N\left(t=T_{0_{-} \text {APАR }}\right)=n-1$

В работе [5] выведена простая формула для расчета средней наработки до отказа АФАР при экспоненциальном распределении отказов СВЧ элементов.

В работах [6,7] исследуется модель надежности ФАР при различных законах распределения отказов СВЧ модулей. Рассматриваются показатели надежности СВЧ модулей ФАР, которые учитывают внезапные и постепенные отказы, для различных законов распределения отказов:

- для экспоненцального распределения (E-R);

- для диффузионного немонотонного распределения (DN-R);

- для распределения Вейбулла (W-R);

- для обобщенного показательного распределения (OP-R);

- для смеси распределений - экспоненциального и Вейбулла (E+W-R).

Критерием отказа ФАР, скомпонованою из $N=n+m$ СВЧ модулей $(n-$ основных та $m$-резервных модулей), является отказ $m+1$ модуля в момент времени $t_{0}=T_{0_{-} \text {APAR }}$

Аналитически условие отказа ФАР формулируется следующим образом

$$
N\left(t_{0}=T_{0 A}\right)=N-m-1
$$

Если $N\left(t_{0}=T_{0 A}\right)=N P_{M}\left(t_{0}=T_{0 A}\right)$, то с учетом выражения (3) формулируется уравнение (4) и (5) для определения средней наработки до отказа ФАР

$$
P_{M}\left(T_{0 A}\right)=1-\frac{m}{N}-\frac{1}{N}
$$

или

$$
\frac{N-m}{N}=P_{M}\left(T_{0 A}\right)
$$

где $P_{M}\left(t_{0}\right)$ - вероятность безотказной работы СВЧ модулей. 
В работах [6,7] проведены исследования поведения вероятности безотказной работы, плотности распределения отказов и интенсивности отказов одноуровневой АФАР при различных законах распределения отказов СВЧ модулей.

В работах [8,9] рассматривается обообщеная вероятностно-физическая модель надежности АФАР с двухуровневой структурной схемой надежности (далее двухуровневая АФАР). В модели надежности двухуровневой АФАР РЛС используется два критерия отказов: критерий допустимого уменьшения количества излучающих каналов в антенной решетке и критерий допустимого повышения минимального уровня боковых лепестков диаграммы направленности амплитудно-фазового распределения антенной решетки. В работах $[8,9]$ выведены уравнения для определения средней наработки до отказа АФАР, получены аналитические выражения для вероятности безотказной работы, плотности распределения вероятности и интенсивности отказов АФАР. Проведены исследования средней наработки до отказа АФАР при экспоненциальном (внезапные отказы) и диффузионном немонотонном (постепенные отказы) распределениях отказов излучающих каналов.

В работе [10] представлена модель надежности передающей АФАР по критерию допустимого снижения максимальной дальности радиолокатора. Выведены трансцендентное уравнение и приближенная формула для определения средней наработки до отказа антенной решетки в зависимости от относительного снижения максимальной дальности РЛС и интенсивностей отказов приемо-передающих каналов и модулей электропитания.

Достоинствами моделей надежности АФАР, представленных в работах [2-10], являются:

- сравнительно простые аналитические соотношения для определения средней наработки до отказа АФАР, практически не зависящие от количества излучающих элементов в антенной решетке;

- критерии отказов АФАР, которые являются ограничениями для основных определяющих параметров в процессе функционирования антенной решетки;

- возможность определения основных показателей надежности и технического обслуживания АФАР: средней наработки до отказа, вероятности безотказной работы, интенсивности отказов, гамма-процентного ресурса и потребного количества запасных каналов и модулей.

Однако применение моделей надежности АФАР РЛС [2-10], возможно при ряде ограничений:

a) для моделей надежности одноуровневой АФАР:

- возможность расчета показателей надежности для АФАР только одного типа: приемной или передающей АФАР;

- возможность использования только одного из критериев отказов: или допустимого снижения максимальной дальности радиолокатора, или допустимого повышения минимального уровня боковых лепестков антенной решетки;

б ) для модели надежности АФАР по критерию допустимого снижения максимальной дальности радиолокатора:

- возможность использования только экспоненциального распределения для отказов антенной решетки и приближенных формул для определения интенсивности отказов АФАР;

- большие погрешности определения вероятности безотказной работы АФАР при использовании экспоненциального распределения отказов (см. рис. 1);

в) для модели надежности двухуровневой АФАР:

- невозможность (напрямую) использования критерия допустимого снижения максимальной дальности локатора для расчета показателей надежности универсальной приемо-передающей АФАР. 


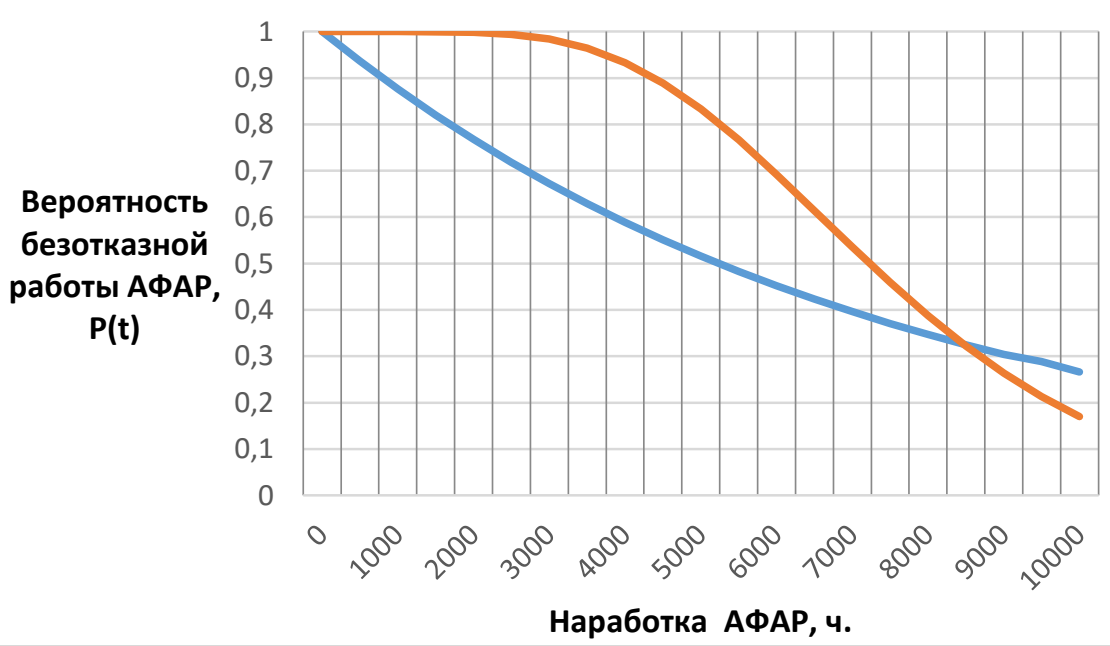

Рис. 1. Графики вероятности безотказной работы АФАР:

для модели надежности по критерию снижения максимальной дальности радиолокатора синяя линия (экспоненциальное распределение отказов), для модели надежности двухуровневой АФАР- красная линия (биноминальное распределение отказов)

\section{3. Постановка проблемы. Цель исследования}

Исходя из изложенного выше в обзоре, для проектирования, производства и эксплуатации многофункциональных РЛС с АФАР является актуальной и необходимой постановка и решение проблемы разработки универсальной модели надежности АФАР, которая бы учитывала два главных физических критерия отказа: снижение максимальной дальности локатора и повышение уровня боковых лепестков диаграммы направленности антенной решетки при отказе излучающих (передающих) и приемных каналов и модулей антенной решетки. Цель исследования - разработка и исследование универсальных моделей надежности АФАР РЛС, учитывающих два главных физических критерия отказа: снижение максимальной дальности локатора и повышение минимального уровня боковых лепестков при отказе излучающих (передающих) и приемных каналов и модулей антенной решетки.

4. Разработка универсальных моделей надежности АФАР РЛС

4.1 Модели отказов приемных и передающих каналов подрешетки и модулей вторичных источников электропитания (табл. 1)

Таблица 1

Модели отказов приемных и передающих каналов и модулей вторичных источников электропитания, учитывающие внезапные и постепенные отказы

\begin{tabular}{|c|c|}
\hline $\begin{array}{l}\text { Тип распределения } \\
\text { отказов }\end{array}$ & Вероятность безотказной работы \\
\hline $\begin{array}{l}\text { Экспоненциальное } \\
\text { распределение (ER) } \\
\text { (внезапные отказы) }\end{array}$ & $P_{E R}(t)=\exp \left(-t / T_{0_{-} M O D_{-} E R}\right)$ \\
\hline $\begin{array}{l}\text { Диффузионное } \\
\text { немонотонное (DNR) } \\
\text { (постепенные отказы) }\end{array}$ & $P_{M O D .}(t)=\Phi\left(\frac{T_{0 \_M O D .}-t}{\sqrt{T_{0 \_M O D .} t}}\right)-\exp (2) \Phi\left(-\frac{T_{0 \_M O D .}+t}{\sqrt{T_{0 \_M O D .} t}}\right)$ \\
\hline $\begin{array}{l}\text { Композиция распре- } \\
\text { делений экспоненциа- } \\
\text { льного и диффузион- } \\
\text { ного немонотонного } \\
\text { (ER и DNR) } \\
\text { (внезапные } \\
\text { постепенные отказы) }\end{array}$ & $P_{M O D_{-} E R^{*} D N R}(t)=\exp \left(-\frac{t}{T_{0_{-} M O D_{-} E R}}\right)\left\{\Phi\left[\frac{T_{0_{-} M O D_{-} D N R}-t}{\sqrt{T_{0_{-} M O D_{-} D N R} t}}\right]-\exp (2) \Phi\left[-\frac{T_{0_{-} M O D_{-} D N R}+t}{\sqrt{T_{0_{-} M O D_{-} D N R} t}}\right]\right\}$ \\
\hline
\end{tabular}


4.2 Универсальная модель надежности передающей АФАР РЛС

Структурная схема надежности передающей двухуровневой АФАР РЛС включает:

- $\mathrm{N}_{\mathrm{R}}$ - общее количество излучающих (передающих) каналов;

- $\mathrm{S}_{\mathrm{R}}$ - количество передающих(излучающих) антенных подрешеток;

- $\mathrm{G}_{\mathrm{R}}$ - количество излучающих каналов в передающей антенной подрешетке;

- $\mathrm{m}_{\mathrm{GR}}$ - допустимое количество отказов излучающих каналов в передающей антенной подрешетке;

- $\mathrm{m}_{\mathrm{SR}}$ - допустимое количество отказов передающих антенных подрешеток в передающей АФАР.

Критерии отказа передающей АФАР:

- первый критерий отказа - допустимое снижение максимальной дальности радиолокатора;

- второй критерий отказа - допустимое количество отказов излучающих антенных подрешеток.

Алгоритм расчета показателей надежности передающей АФАР включает три последовательных шага:

\section{Первый шаг:}

Определение значения средней наработки до отказа передающей $\mathrm{AФAР} T_{0_{-} A P A R_{-}}$, по критерию допустимого снижения максимальной дальности локатора из решения трансцендентного уравнения (9) [10]:

$$
\left(1-\frac{\Delta D}{D}\right)^{4}=9-2 P_{R A D_{-} A N_{-} M}\left(T_{0_{-} A P A R}\right)-2 P_{R_{A D} M_{-} M_{-} P A L A R}\left(T_{0_{-} A P A R}\right)-3 P_{S P S}\left(T_{0_{-} A P A R}\right),
$$

где $P_{R A D_{-} A N_{-} M}\left(T_{0_{-} A P A R}\right)$ - вероятность безотказной работы излучающего (передающего) канала антенной решетки;

$P_{R A D_{-} M_{-} S P A L A R}\left(T_{0 \_A P A R}\right)$ - вероятность безотказной работы излучающего (передающего) канала модуля антенной подрешетки;

$P_{S P S}\left(T_{0_{-} A P A R}\right)$ - вероятность безотказной работы модуля вторичного источника питания;

$\frac{\Delta D}{D}$ - допустимое снижение максимальной дальности радиолокатора.

\section{Второй шаг:}

Рассчитывается допустимое число отказов антенных передающих подрешеток $\mathrm{m}_{\mathrm{SR}}$ двухуровневой АФАР (которое приближенно обеспечивает значение средней наработки до отказа $T_{0_{-} A P A R_{-}}$, определенное на первом шаге) и значение средней наработки до отказа передающей АФАР $T_{0_{-} A P A R_{-} 2}$, (используя модель надежности двухуровневой АФАР) из решения уравнения:

$$
P_{S P S}\left(t=T_{0_{-} R A D . A P A R}\right) P_{R A D . S L A P A R}\left(t=T_{0_{-} R A D . A P A R}\right)=1-C_{2}-\frac{1}{S_{0}},
$$

где вероятность безотказной работы передающей подрешетки определяется по формуле

$$
P_{\text {RAD.SLAPAR. }}(t)=P_{S P S}(t) \sum_{i=0}^{m_{\text {RAD.CHAN. }}} C_{G_{0}}^{i}\left[P_{\text {RAD.CHAN. }}(t)\right]^{G_{0}-i}\left[Q_{\text {RAD.CHAN. }}(t)\right]^{i},
$$

\section{Примечание:}

При расчете $T_{0_{-} A P A R_{-} 2}$ двухуровневой АФАР принимается условие, что $m_{G R}=2 G_{R} \times \frac{\Delta D}{D}$ 


\section{Третий шаг:}

Используя значение средней наработки до отказа $T_{0_{-} A P A R_{-} 2}$ и значение допустимого количества отказов антенных передающих подрешеток $\mathrm{m}_{\mathrm{S} R}$ двухуровневой АФАР, определяются вероятность безотказной работы, интенсивность отказов и другие показатели надежности передающей АФАР:

$$
P_{R A D . A P A R}(t)=\sum_{i=0}^{m_{R A D . S L A P A R}} C_{S_{0}}^{i}\left[P_{R A D . S L A P A R}(t)\right]^{S_{0}-i}\left[Q_{R A D . S L A P A R}(t)\right]^{i},
$$

\section{2 Универсальная модель надежности приемной АФАР РЛС}

Структурная схема надежности приемной АФАР РЛС включает:

$\mathrm{N}_{\mathrm{P}}$ - общее количество приемных каналов;

$\mathrm{S}_{\mathrm{P}}$ - количество приемных антенных подрешеток;

$\mathrm{G}_{\mathrm{P}}$ - количество приемных каналов в приемной антенной подрешетке;

$\mathrm{m}_{\mathrm{GP}}$ - допустимое количество отказов приемных каналов в приемной антенной подрешетке;

$\mathrm{m}_{\mathrm{SP}}$ - допустимое количество отказов приемных антенных подрешеток в приемной АФАР

Критерии отказа приемной АФАР:

- первый критерий отказа - допустимое снижение максимальной дальности радиолокатора;

- второй критерий отказа - допустимое количество отказов приемных антенных подрешеток.

Алгоритм расчета показателей надежности приемной АФАР включает два последовательных шага:

\section{Первый шаг:}

Используя модель надежности двухуровневой АФАР, для допустимого количества отказов антенных подрешеток $\mathrm{m}_{\mathrm{SP}}$ (допустимого повышения минимального уровня боковых лепестков диаграммы направленности) из решения трансцендентного уравнения (13) рассчитывается значение средней наработки до отказа $T_{0_{-} A P A R_{-} R E C}$ приемной АФАР:

$$
P_{\text {REC.MOD. }}\left(t=T_{0_{-} \text {REC.APAR }}\right) P_{S P S}\left(t=T_{0_{-} R E C . A P A R}\right) P_{R E C . S L A P A R}\left(t=T_{0_{-} R E C . A P A R}\right)=1-C_{1}-\frac{1}{S_{0}}
$$

и вероятности безотказной работы приемной подрешетки по формуле:

$$
P_{\text {RES.SLAPAR. }}(t)=P_{\text {REC.MOD. }}(t) P_{S P S}(t) \sum_{i=0}^{m_{\text {REC.CHAN. }}} C_{G_{0}}^{i}\left[P_{\text {REC.CHAN. }}(t)\right]^{G_{0}-i}\left[Q_{\text {REC.CHAN. }}(t)\right]^{i},
$$

\section{Примечание:}

При расчете $T_{0_{-} A P A R_{-} R E C}$ двухуровневой АФАР принимается условие, что $m_{G R}=G_{R} \times \frac{\Delta D}{D}$

\section{Второй шаг:}

Используя значение средней наработки до отказа $T_{0_{-} A P A R_{-} R E C}$ И значение допустимого количества отказов антенных приемных подрешеток $\mathrm{m}_{\mathrm{SP}}$ двухуровневой АФАР, определяются вероятность безотказной работы, интенсивность отказов и другие показатели надежности приемной АФАР:

$$
P_{R E C . A P A R}(t)=\sum_{i=0}^{m_{R E C . S L A P A R}} C_{S_{0}}^{i}\left[P_{R E S . S L A P A R}(t)\right]^{S_{0}-i}\left[Q_{R E C . S L A P A R}(t)\right]^{i},
$$

Универсальная модель надежности приемо-передающей АФАР РЛС 
Структурная схема надежности приемо-передающей двухуровневой АФАР РЛС включает:

$\mathrm{N}$ - общее количество приемных и передающих каналов;

$\mathrm{S}$ - количество приемо-передающих антенных подрешеток;

$\mathrm{G}$ - количество приемных и передающих каналов в приемо-передающей антенной подрешетке;

$\mathrm{m}_{\mathrm{G}}$ - допустимое количество отказов приемных и передающих каналов в приемопередающей антенной подрешетке;

$\mathrm{m}_{\mathrm{S}}$ - допустимое количество отказов приемо-передающих антенных подрешеток в приемо-передающей АФАР.

Критерии отказа приемо-передающей АФАР:

- первый критерий отказа - допустимое снижение максимальной дальности радиолокатора;

- второй критерий отказа - допустимое количество отказов приемо-передающих антенных подрешеток, определяемых допустимым повышением минимального уровня боковых лепестков диаграммы направленности

Алгоритм расчета показателей надежности приемо-передающей АФАР включает четыре последовательных шага:

Первый шаг:

Определение значения средней наработки до отказа $T_{0_{-} A P A R_{-}}$, передающей АФАР по критерию допустимого снижения максимальной дальности локатора из решения трансцендентного уравнения (9):

\section{Второй шаг:}

Рассчитывается значение допустимого количества отказов антенных передающих подрешеток $\mathrm{m}_{\mathrm{SR}}$ двухуровневой AФAP (которое обеспечивает (приближенно) значение средней наработки до отказа $T_{0_{-} A P A R}{ }_{-}$определенное на первом шаге) и соответствующее ему значение средней наработки до отказа $T_{0_{-} A P A R_{-} 2}$, передающей части АФАР, используя модель надежности двухуровневой АФАР, из решения уравнения

$$
P_{S P S}\left(t=T_{0_{-} R A D . A P A R}\right) P_{R A D . S L A P A R}\left(t=T_{0_{-} R A D . A P A R}\right)=1-C_{2}-\frac{1}{S_{0}}
$$

где вероятность безотказной работы передающей подрешетки вычисляется формуле:

$$
P_{R A D . S L A P A R .}(t)=P_{S P S}(t) \sum_{i=0}^{m_{R A D . C H A N .}} C_{G_{0}}^{i}\left[P_{R A D . C H A N .}(t)\right]^{G_{0}-i}\left[Q_{R A D . C H A N .}(t)\right]^{i}
$$

Примечание: При расчете $T_{0_{-} A P A R_{-} 2}$, двухуровневой АФАР принимается условие, что $m_{G R}=2 G_{R} \times \frac{\Delta D}{D}$

\section{Третий шаг:}

Используя модель надежности двухуровневой АФАР, для допустимого количества отказов антенных подрешеток $\mathrm{m}_{\mathrm{SP}}$ (допустимого повышения минимального уровня боковых лепестков диаграммы направленности) из решения транцендентного уравнения (13) рассчитывается соответствующее ему значение средней наработки до отказа $T_{0_{-} A P A R_{-} R E C}$, приемной части АФАР.

Примечание: При расчете $T_{0_{-} A P A R_{-} R E C}$, двухуровневой АФАР принимается условие, что

$$
m_{G R}=G_{R} \times \frac{\Delta D}{D} .
$$




\section{Четвертый шаг:}

Используя значение средней наработки до отказа $T_{0_{-} A P A R_{-} 2}$, и значение допустимого количества отказов приемо-передающих антенных подрешеток $\mathrm{m}_{\mathrm{S}}$ двухуровневой АФАР, определяются вероятность безотказной работы, интенсивность отказов и другие показатели надежности приемной и передающих частей АФАР по формулам (12) и (15).

5. Иллюстративные примеры определения показателей надежности приемной, передающей и приемо-передающей АФАР при использовании универсальных моделей надежности

\section{1 Пример расчета показателей надежности передающей АФАР}

Структурная схема надежности передающей двухуровневой АФАР РЛС включает:

$\mathrm{N}_{\mathrm{R}}=6400$ передающих (излучающих) каналов; $\mathrm{S}_{\mathrm{R}}=100$ передающих (излучающих) антенных подрешеток; $\mathrm{G}_{\mathrm{R}}=64$ передающих (излучающих) каналов в передающей антенной подрешетке; $\mathrm{m}_{\mathrm{GR}}=13$ допустимое количество отказов излучающих каналов в передающей антенной подрешетке; $\mathrm{m}_{\mathrm{SR}}$ - допустимое количество отказов передающих антенных подрешеток в передающей АФАР.

Отказы передающих каналов и модулей распределяются по экспоненциальному закону (внезапные отказы).

Интенсивности отказов передающих (излучающих) каналов антенных модулей $\lambda_{\text {RAD.AN.M. }}$ изменяются в диапазоне от $0,000004 ч^{-1}$ до 0,000013 ч $^{-1}$. Интенсивность отказов. передающих (излучающих) каналов модулей антенной подрешетки $\lambda_{\text {RAD.M.SPALAR }}=0,000001 ч^{-1}$.

Интенсивности отказов модулей вторичного электропитания $\lambda_{\text {SPS }}$ изменяются в диапазоне от $0,000005 \mathrm{ч}^{-1}$ до $0,000020 \mathrm{ч}^{-1}$. Допустимое количество отказов передающих антенных подрешеток в передающей АФАР $\mathrm{m}_{\mathrm{SR}}$, изменяется от 1 до 10.

На рис. 2 представлены графики зависимостей средней наработки до отказа передающей АФАР от допустимого снижения максимальной дальности локатора $\Delta \mathrm{D} / \mathrm{D}$ для одноуровневой АФАР (при расчете по критерию допустимого снижения максимальной дальности локатора (красная линия)) и для двухуровневой АФАР (по критерию допустимого снижения количества излучающих каналов в антенной решетке (синяя линия)).

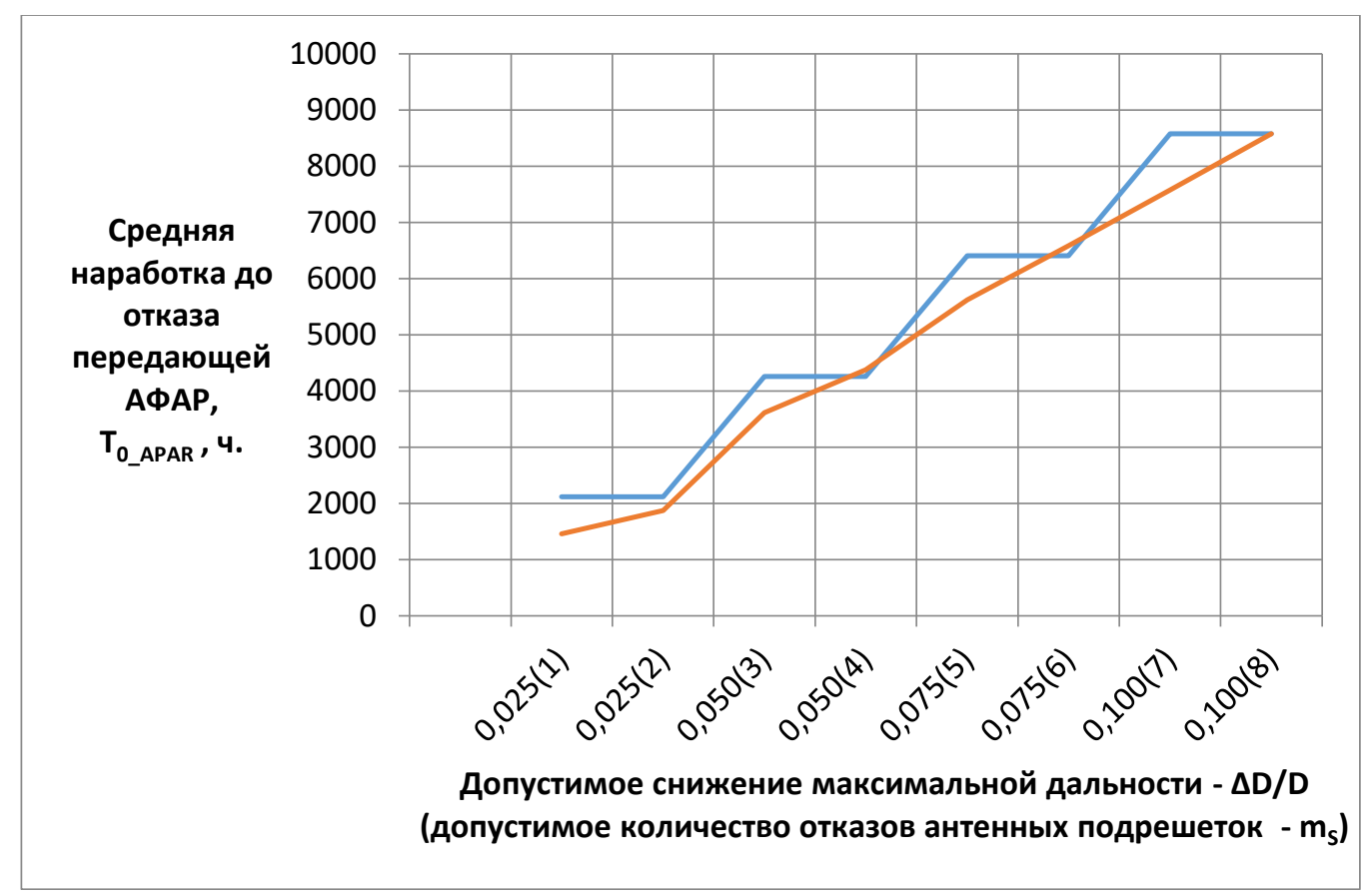

Рис. 2. Графики зависимости средней наработки до отказа передающей АФАР для двух критериев отказов 
На рис. 2 допустимое количество отказов передающих антенных подрешеток двухуровневой АФАР зависит от допустимого снижения максимальной дальности локатора $\Delta \mathrm{D} / \mathrm{D}$ и изменяется по следующей схеме: для $\Delta \mathrm{D} / \mathrm{D}=0,025-\mathrm{m}_{\mathrm{SR}}=1,2$; для $\Delta \mathrm{D} / \mathrm{D}=0,050$ $\mathrm{m}_{\mathrm{SR}}=3,4 ;$ для $\Delta \mathrm{D} / \mathrm{D}=0,075-\mathrm{m}_{\mathrm{SR}}=5,6 ;$ для $\Delta \mathrm{D} / \mathrm{D}=0,100-\mathrm{m}_{\mathrm{SR}}=7,8$.

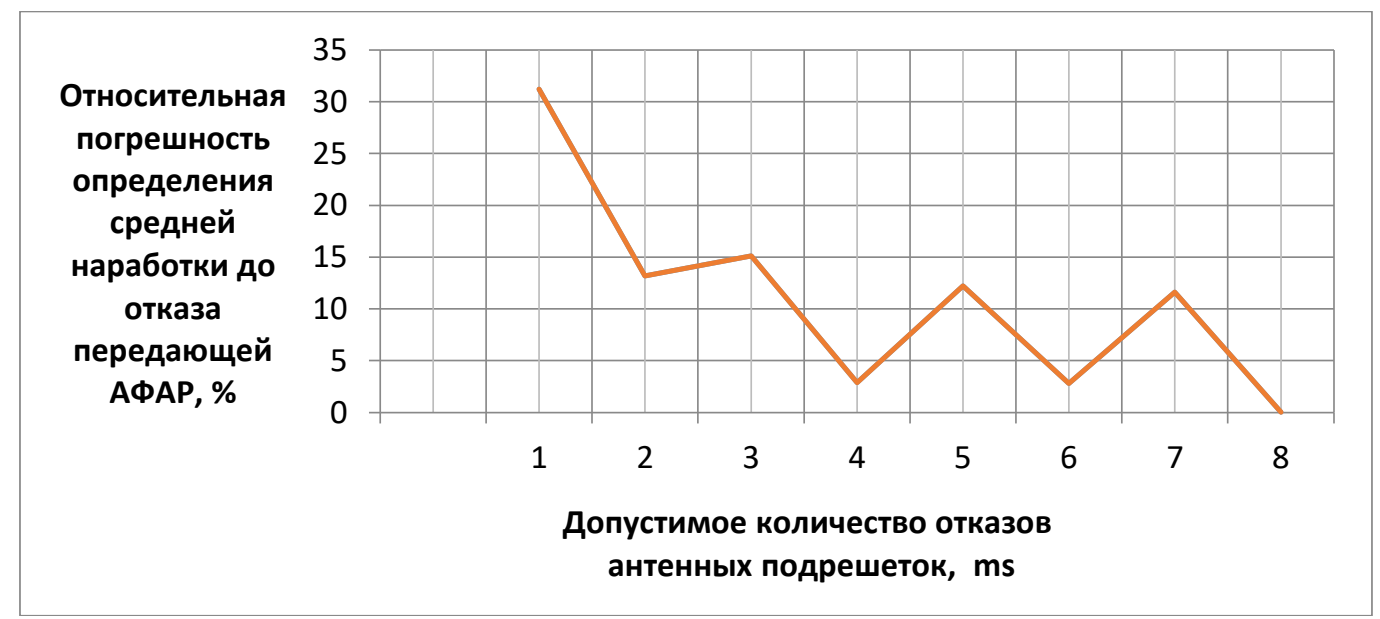

Рис. 3. График зависимости относительной погрешности определения средней наработки до отказа передающей АФАР от допустимого количества отказов антенных подрешеток

Как видно из рис. 3 относительная погрешность определения значения средней наработки до отказа передающей АФАР для двухуровневой АФАР (по сравнению с двухуровневой АФАР) составляет от 0 до 15\%, что вполне удовлетворяет условию проведения расчетов надежности АФАР в пределах инженерной точности.

На рис. 4 представлены графики зависимости средней наработки до отказа передающей АФАР $T_{0_{-} \text {AРАR_1 }}$, модель одноуровневой АФАР) от интенсивностей отказов передающих каналов антенных модулей при различных значениях интенсивностей отказов модулей электропитания.

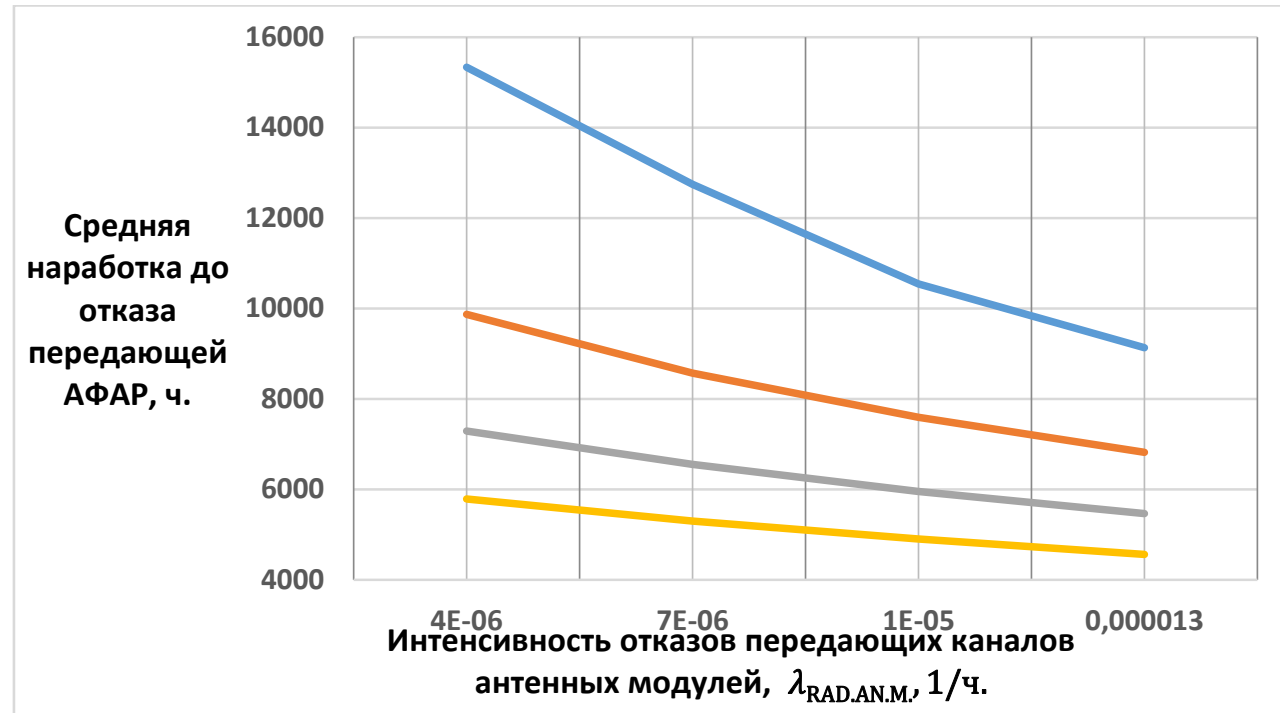

Рис.4. Графики зависимости средней наработки до отказа передающей одноуровневой АФАР и передающей части приемо-передающей $\mathrm{AФAР} \mathrm{для} \Delta \mathrm{D} / \mathrm{D}=0,100$ от интенсивностей отказов передающих каналов антенных модулей

На рис.4 наведено: при при различных интенсивностях отказов модулей вторичного электропитания $\lambda_{\mathrm{ME}}=0,0000051 /$ ч. - линия синего цвета; при $\lambda_{\mathrm{ME}}=0,0000101 /$ ч. - линия 
красного цвета; при $\lambda_{\mathrm{ME}}=0,0000151 /$ ч. - линия зеленого цвета; при $\lambda_{\mathrm{ME}}=0,0000201 /$ ч. линия фиолетового цвета

На рис. 5 представлены графики зависимости средней наработки до отказа передающей АФАР $T_{0 \_A P A R_{-} 2}$, (модель двухуровневой АФАР) от интенсивностей отказов передающих каналов антенных модулей для допустимого снижения максимальной дальности радиолокатора $\Delta \mathrm{D} / \mathrm{D}=0,100$ при различных значениях допустимых отказов передающих антенных подрешеток.

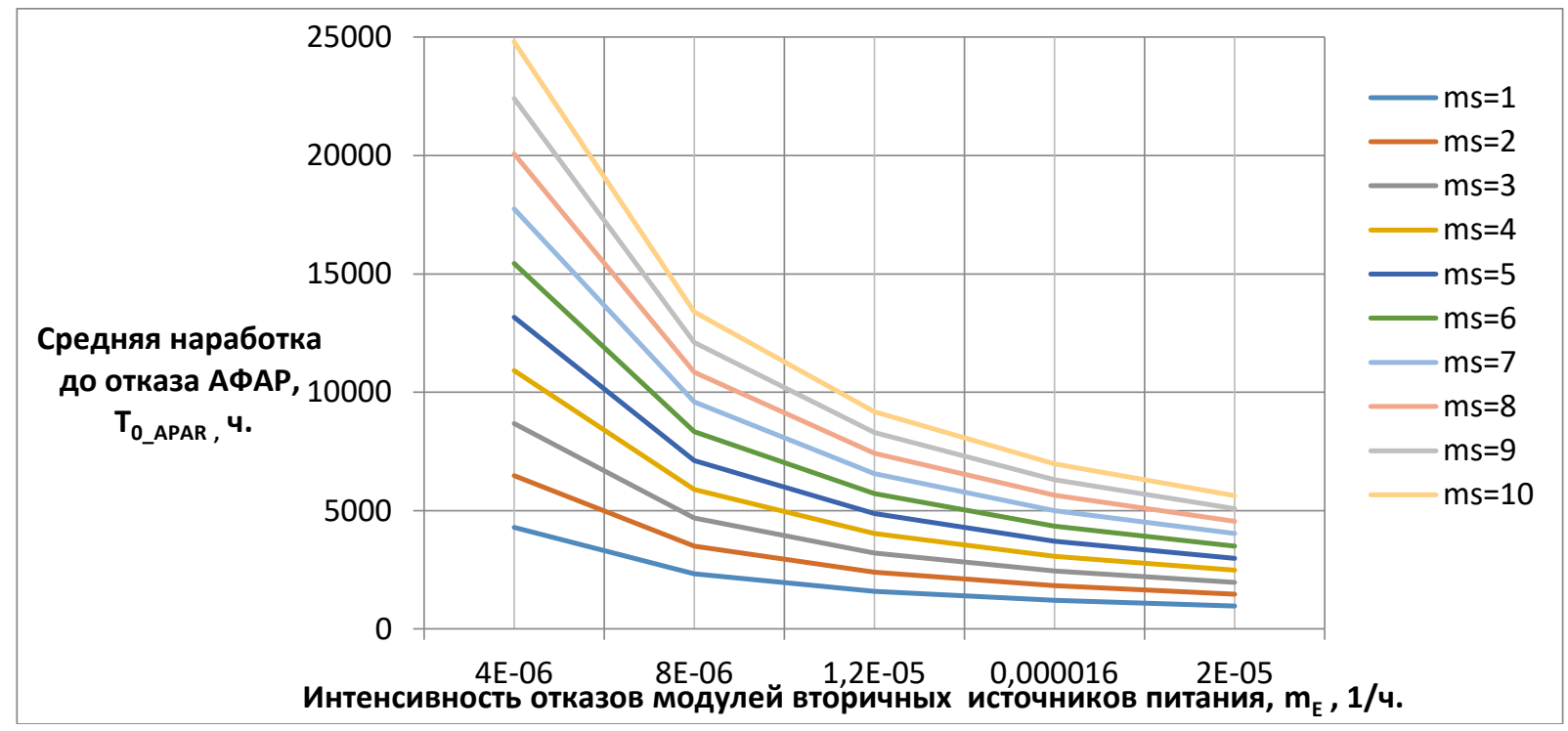

Рис. 5. Графики зависимости средней наработки до отказа передающей AФАР T $0 \_A P A R$ OT интенсивностей отказов модулей вторичного электропитания $\lambda_{\mathrm{ME}}$ для $\Delta \mathrm{D} / \mathrm{D}=0,100$ при различных значениях допустимого количества отказов антенных подрешеток $\mathrm{m}_{\mathrm{SR}}$

\section{2 Пример расчета показателей надежности приемной АФАР РЛС}

Структурная схема надежности приемной АФАР РЛС включает:

$\mathrm{N}_{\mathrm{P}}=6400$ - общее количество приемных каналов; $\mathrm{S}_{\mathrm{P}}=100$ - количество приемных антенных подрешеток; $\mathrm{G}_{\mathrm{P}}=64$ - количество приемных каналов в приемной антенной подрешетке; $\mathrm{m}_{\mathrm{GP}}=6$ - допустимое количество отказов приемных каналов в приемной антенной подрешетке; $\mathrm{m}_{\mathrm{SP}}$ - допустимое количество отказов приемных антенных подрешеток в приемной АФАР.

Отказы приемных каналов и модулей электропитания распределяются по экспоненциальному закону (внезапные отказы).

Интенсивности отказов приемных каналов антенных модулей: $\lambda_{\text {REC.AN.M. }}=0,0000007$ ч $^{-1}$. Интенсивность отказов. приемных каналов модулей антенной подрешетки: $\lambda_{\text {REC.M.SPALAR }}=$ $0,0000007 ч^{-1}$. Интенсивности отказов модулей вторичного электропитания $\lambda_{\text {SPS }}$ диапазоне от $0,000005 ч^{-1}$ до $0,000020 ч^{-1}$.

Допустимое количество отказов приемных антенных подрешеток в приемной АФАР m $\mathrm{SP}$, изменяется от 1 до 5. На рис. 6 представлены графики зависимостей средней наработки до отказа приемной АФАР от интенсивностей отказов модулей вторичного электропитания при различных значениях допустимого количества отказов приемных антенных подрешеток.

\section{3 Пример расчета показателей надежности приемо-передающей АФАР РЛС}

Исходные данные раздела 5.3 соответствуют исходным данным разделов 5.1 и 5.2, за исключением количества допустимых отказов приемных каналов антенных модулей: $\mathrm{m}_{\mathrm{GP}}=$ 13.

Результаты расчетов показывают, что показатели надежности передающей части приемопередающей АФАР соответствуют показателям надежности передающей АФАР (раздел 5.1), a показатели надежности приемной части приемо-передающей АФАР соответствуют 
показателям надежности приемной АФАР (раздел 5.2). Для приемо-передающей АФАР в целом надежность будут определяться показателями надежности приемной АФАР, так как они имеют более низкие значения по сравнению с показателями надежности передающей AФАР.

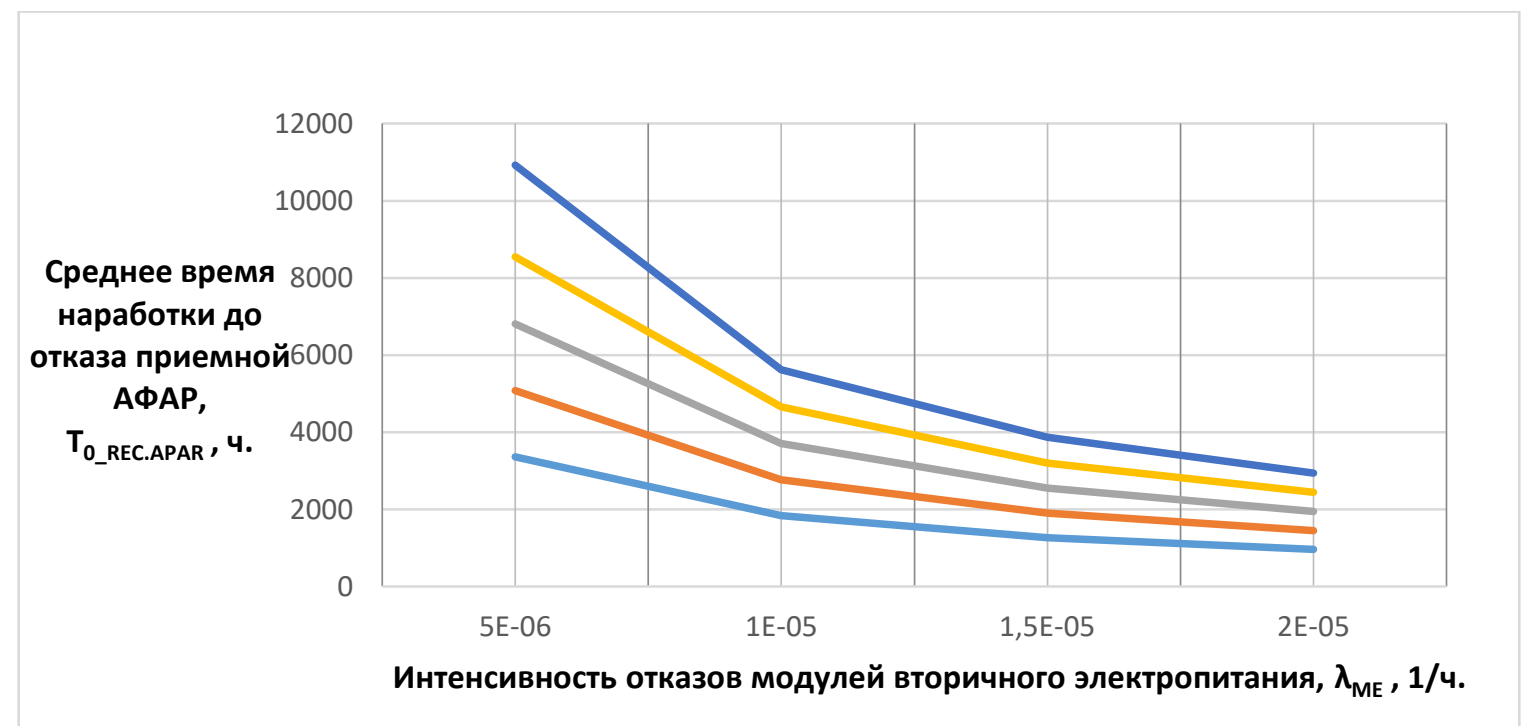

Рис. 6. Графики зависимости средней наработки до отказа приемной АФАР Т интенсивностей отказов модулей вторичного электропитания $\lambda_{\mathrm{ME}}$ при различных значениях допустимого количества отказов антенных подрешеток $\mathrm{m}_{\mathrm{S}}$ :

при $\mathrm{m}_{\mathrm{SP}}=1$ - синяя линия; при $\mathrm{m}_{\mathrm{SP}}=2$ - красная линия; при $\mathrm{m}_{\mathrm{SP}}=3$ - зеленая линия; при $\mathrm{m}_{\mathrm{SP}}=4$ - фиолетовая линия; при $\mathrm{m}_{\mathrm{SP}}=5$ - голубая линия

\section{6. Выводы}

6.1 Проведен анализ и систематизация моделей надежности АФАР РЛС, разработанных за последние 20 лет.

6.2 Модель надежности АФАР РЛС по критерию допустимого снижения минимальной дальности локатора может служить приближенной моделью для определения средней наработки до отказа передающей АФАР.

6.3 Модель двухуровневой АФАР, учитывающая два критерия отказов: допустимое снижение количества излучаемых модулей и допустимое снижение количества приемных подрешеток, является основой для построения универсальных моделей надежности для передающей, приемной и приемо-передающей АФАР.

6.4 Универсальные модели надежности АФАР позволяют определить следующие показатели надежности антенной решетки: среднюю наработку до отказа, вероятность безотказной работы, интенсивность отказов, гамма-процентные ресурсы и др.

6.5 Средняя наработка до отказа передающей и приемо-передающей АФАР в значительной мере зависит от значений интенсивностей отказов излучающих (приемных) каналов и источников вторичного питания.

6.6 Средняя наработка до отказа приемной АФАР в значительной мере зависит от интенсивностей отказов источников вторичного питания.

6.7 Предлагаемые в данной статье универсальные модели надежности АФАР рекомендуется использовать при проектировании новых многофункциональных РЛС с АФАР и разработке их системы технического обслуживания. 


\section{ЛИТЕРАТУРА}

1. Пак Я., Стрелецкий А. Развитие систем ПВО-ПРО боевых кораблей ВМС США [Текст]: / М. : Зарубежное военное обозрение.- 2017 - №5- С. 66-75.

2. Ashok K. Agrawal Active Phased Array Design for High Reliability, [Text] / Ashok K. Agrawal and Eric L. Holzman // IEEE Transactions on aerospace and electronic systems - Vol. 35 - NO. 4 - October 1999 - p.p. 1204 -1211.

3. Ashok K. Agrawal. Beamformer Architectures for Active Phased-Array Radar Antennas, [Text] / Ashok K. Agrawal, and Eric L. Holzman // IEEE Transactions on antennas and propagation Vol. 47 - No. 3 -march 1999 - p.p. 432 - 442.

4. Ashok K. Agrawal. Active Phased Array Antenna Development for Modern Shipboard Radar Systems , [Text] / Ashok K. Agrawal, Bruce A. Kopp, Mark H. Luesse, and Kenneth W. O'Haver //Active phased array antenna development johns Hopkin sapl technical direct Volume 22 /// - Number 4 (2001) - p.p. 600-612.

5. Kostanovskii V. V. A mathematical model for calculating the reliability of nonreducible phased antenna arrays, [Text] / Kostanovskii V.V. //Measurement Techniques - 2014 -T. 57 - № 1. /// M.: C. 87-90.

6. Костановський В. В. Математичні моделі надійності типових апертур фазованих антенних решіток, які враховують раптови та поступові відмови модулів надвисоких частот. / Науковий журнал: Математичні машини і системи - // - НАНУ, 2014 р. № 2 $142-150$ c.

7. Костановский В. В., Козачук О. Д. Вероятностный анализ безотказности и долговечности апертур фазированных антенных решеток в процессе проектирования. [Текст]: / Науковий журнал: Математичні машини і системи. // - НАНУ, 2015 р. №3. С. 201-213 с.

8. V. Kostanovskyi, I. Machalin, O. Kozachuk, I. Terentyeva. Construction of a generalized probabilistic-physical model of reliability of two-level active phased antenna array / EasternEuropean journal of enterprise technologies, 3/9 (99) 2019/ УДК.623.6-523.8:6234 DOI:10.15587/1729-4061.2019.168525, стр. 31-40.

Построение обобщенной вероятностно-физической модели надежности двухуровневой активной фазированной антенной решетки В. В. Костановский, И. А. Мачалин, О. Д. Козачук, И. А. Терентьева.

9. Костановський В. В. Визначення області існування показників надійності в залежності від допустимих значень показників єфектівності активной фазованої антенної решіткі / Науковий журнал: Математичні машини і системи - // - НАНУ, 2019 р. № 4, 154 - 164 с.

10. Костановський В. В., Демченко О. В., Козачук О. Д., Мачалін І. О. Модель розрахунку показників надійності АФАР за критерієм допустимого зниження максімальної дальності РЛС [Текст]/ Науковий журнал: Наукоємні технології, №1, 2020 p.

Костановский В.В., Мачалин И.А.

РАЗРАБОТКА И ИССЛЕДОВАНИЕ УНИВЕРСАЛЬНЫХ МОДЕЛЕЙ НАДЕЖНОСТИ АКТИВНОЙ ФАЗИРОВАННОЙ МНОГОФУНКЦИОНАЛЬНОЙ РАДИОЛОКАЦИОННОЙ СТАНЦИИ

В работе проведен краткий анализ моделей надежности АФАР, предложенными учеными и инженерами за последние 20 лет. Рассмотрена модель надежности приемной АФАР по критерию допустимого повымения минимального уровня боковых лепестков диаграммы направленности амплитудно-фазового распределения антенной решетки. Проанализирована модель надежности передающей АФАР по критерию допустимого снижения максимальной дальности РЛС. Показано, что рассмотренные модели надежности являются приближенными (используют экспоненциальный закон распределения отказов АФАР) и поэтому могут использоваться только для приближенного определения средней наработки до отказа антенной решетки. Для определения вероятностных показателей надежности АФАР: вероятности безотказной работы, интенсивности 
отказов, плотности распределения отказов, гамма-прочентных ресурса и др. необходимы более универсальные модели надежности, учитывающие структуру и сложность построения антенной решетки как системы, состоящей из огромного количества основных и резервных приемо-передающих каналов и модулей. В работе разработаны и исследовань универсальные модели надежности АФАР многофункииональной РЛС $с$ двухуровневой структурной схемой надежности (двухуровневая АФАР). Универсальные модели надежности учитывает два основных физических критерия отказа АФАР: допустимое снижение максимального уровня дальности РЛС и допустимое увеличение минимального уровня боковых лепестков диаграммы направленности антенной решетки. $B$ качестве законов распределения отказов излучающих (передающих) и приемных каналов и модулей антенной решетки применяется экспоненциальное распределение (характеризует внезапные отказы), немонотонное диффузионное распределение (характеризует постепенные отказы) и композиция экспоненциального и диффузионного немонотонного закона распределений (характеризует совместное проявление внезапных и постепенных отказов). В статье представлень уравнения и формуль для определения показателей надежности передающей, приемной и приемно-передающей АФАР: средней наработки до отказа, вероятности безотказной работь $u$ др. $B$ работе рассмотрено три иллюстративных примера расчета показателей надежности передающей, приемной $u$ приемо-передающей АФАР при экспоненциальном распределении отказов каналов и модулей антенной решетки. Проведен анализ влияния интенсивностей отказов излучаюших каналов и модулей источников вторичного питания на среднюю наработку до отказа АФАР.

Представленные в статье универсальные модели надежности АФАР рекомендуется использовать при разработке новых многофункциональных РЛС с АФАР, а также могут быть полезны студентам и аспирантам университетов и ВУЗов в учебном процессе.

Ключевые слова: показатели надежности, средняя наработка до отказа, активная фазированная антенная решетка, передающие и приемные каналы антенной решетки.

\section{Kostanovskiy V., Machalin I. \\ DEVELOPMENT AND RESEARCH OF UNIVERSAL RELIABILITY MODELS OF AN ACTIVE PHASED ANTENNA ARRAY OF A MULTIFUNCTIONAL RADAR STATION}

The paper provides a brief analysis of the AFAR reliability models proposed by scientists and engineers over the past 20 years. The reliability model of the receiving AFAR is considered according to the criterion of permissible increase in the minimum level of the side lobes of the radiation pattern of the amplitude-phase distribution of the antenna array. The reliability model of the transmitting AFAR is analyzed according to the criterion of the permissible decrease in the maximum radar range. It is shown that the considered reliability models are approximate (they use the exponential distribution law of AFAR failures) and therefore can only be used to approximate the mean time between failure of the antenna array. To determine the probabilistic indicators of AFAR reliability: the probability of failure-free operation, the failure rate, the distribution density of failures, the gamma-percent resource, etc. more universal reliability models are needed that take into account the structure and complexity of constructing an antenna array as a system consisting of a huge number of primary and backup receivers transmitting channels and modules. In the work, universal reliability models of the AFAR reliability of a multifunctional radar with a two-level structural reliability scheme (two-level AFAR) were developed and investigated. Universal reliability models take into account two basic physical criteria of AFAR failure: an acceptable decrease in the maximum level of radar range and an acceptable increase in the minimum level of side lobes of the antenna array radiation pattern. As the laws of distribution of failures of the emitting (transmitting) and receiving channels and modules of the antenna array, an exponential distribution (characterizes sudden failures), a nonmonotonic diffusion distribution (characterizes gradual failures), and a composition of the exponential and diffusive nonmonotonic distribution law (characterizes the joint manifestation of sudden and gradual failures). The article presents 
equations and formulas for determining the reliability indicators of a transmitting, receiving and receiving-transmitting AFAR: the average operating time to failure, the probability of failure-free operation, etc. Three illustrative examples of calculating the reliability indicators of a transmitting, receiving, and transmitting AFAR with an exponential distribution are considered channel failures and antenna array modules. The analysis of the effect of the failure rates of the emitting channels and the modules of the secondary power sources on the mean time between failures of the AFAR is performed. The universal AFAR reliability models presented in the article are recommended to be used when developing new multifunctional radars with AFAR, and can also be useful to students and graduate students of universities and universities in the educational process.

Key words: reliability indicators, mean time between failures, active phased antenna array, transmitting and receiving channels of the antenna array. 